\title{
Association between STAT4 gene polymorphism and type 2 diabetes risk in Chinese Han population
}

Jiaqi Cui ${ }^{1 \dagger}$, Rui Tong ${ }^{1 \dagger}$, Jing Xu' ${ }^{1}$, Yanni Tian², Juan Pan ${ }^{3}$, Ning Wang ${ }^{1}$, Huan Chen ${ }^{1}$, Yanqi Peng ${ }^{1}$, Sijia Fei ${ }^{1}$, Wang Ling ${ }^{1}$, Chaoying Guo ${ }^{1}$, Juanchuan Yao ${ }^{1}$ and Wei Cui ${ }^{\text {* }}$

\begin{abstract}
Background: Evidence from genetic epidemiology indicates that type 2 diabetes (T2D) has a strong genetic basis. Activated STAT4 has an inflammatory effect, and STAT4 is an important mediator of inflammation in diabetes. Our study aimed to study the association between STAT4 single nucleotide polymorphisms (SNPs) and T2D susceptibility in Chinese Han population.
\end{abstract}

Methods: We conducted a 'case-control'study among 500 T2D patients and 501 healthy individuals. 5 candidate STAT4 SNPs were successfully genotyped. The association between SNPs and T2D susceptibility under different genetic models was evaluated by logistic regression analysis. 'SNP-SNP' interaction was analyzed and completed by multi-factor dimensionality reduction (MDR). Finally, we evaluated the differences of clinical characteristics under different genotypes by one-factor analysis of variance.

Results: The overall results showed that STAT4 rs3821236 was associated with increasing T2D risk under allele (OR $1.23, p=0.020$ ), homozygous (OR 1.51, $p=0.025$ ), dominant (OR 1.36, $p=0.029$ ), and additive models (OR 1.23, $p=0.020$ ). The results of stratified analysis showed that rs3821236, rs 11893432, and rs 11889341 were risk factors for T2D among participants $\leq 60$ years old. Only rs 11893432 was associated with increased T2D risk among female participants. There was also a potential association between rs3821236 and T2D with nephropathy risk. STAT4 rs11893432, rs7574865 and rs897200 were significantly associated with lysophosphatidic acid, cystatin C and thyroxine 4 , respectively.

Conclusion: The genetic polymorphisms of STAT4 is potentially associated with T2D susceptibility of Chinese population. In particular, rs3821236 is significantly associated with T2D risk both in the overall and several subgroup analyses. Our study may provide new ideas for T2D individualized diagnosis/protection.

Keywords: Diabetes, STAT4, Single nucleotide polymorphism, Chinese Han population

*Correspondence: doctorweiwei.cui@126.com

${ }^{\dagger}$ Jiaqi Cui and Rui Tong: Co-first author

${ }^{1}$ Department of Endocrinology and Second Department of Geriatrics, The First Affiliated Hospital of Xi'an Jiaotong University, \#277 West Yanta Road, Xi'an 710061, Shaanxi, China

Full list of author information is available at the end of the article

\section{Background}

Diabetes is a disease of various metabolic disorders caused by impaired glucose metabolism characterized by hyperglycemia $[1,2]$. The study found that with the gradual passage of time, the age of onset of diabetes tends to be younger [3]. Its incidence rate has increased year by year and diabetes has become an important public health problem globally. At present, China has become the 
second largest country in the world after India in terms of number of diabetic patients. It is estimated that the total number of diabetes patients in China will be close to 100 million by 2025 [4]. According to previous reports, it is generally believed that diabetes is often caused by the interaction of genetic and environmental factors resulting in insufficient insulin secretion. Evidence from genetic epidemiology indicates that the onset of type 2 diabetes has a strong genetic basis, and its genetic model belonged to polygenetics [5]. In recent years, with the development of molecular biology and molecular epidemiology and the improvement and application of gene detection technology, some genetic polymorphism loci associated with type 2 diabetes have been identified [6]. Up to now, T2D risk assessments have been conducted only in some populations. Therefore, it is still a difficult task to discover genetic polymorphism loci associated with T2D risk-among populations with different genetic backgrounds.

STAT4 is expressed in immunoregulatory cells such as monocytes, dendritic cells, and macrophages at the site of inflammation. STAT4 mainly induces Th1 responses and inhibits Th2responses [7, 8]. Activated STAT4 is considered to have inflammatory effect, it plays an important role in the regulation of $\mathrm{Th} 1 / \mathrm{Th} 2$ differentiation and the autoimmune diseases caused by this disorder. STAT4 is an important mediator of inflammation in immune cells and fat cells in diabetes and obesity [9]. More importantly, several studies have found Th1/Th2 cytokine imbalance in T2D patients [10-12], we speculate that STAT4 gene may play a potential role in the occurrence and development of type 2 diabetes. STAT4 genetic polymorphisms associated with the development of various diseases have been reported [13-19]. We did not find any reports on the association between STAT4 genetic polymorphisms and T2D risk.

Therefore, this study took the Chinese Han population as the research object and selected 5 candidate STAT4 SNPs (rs3821236 A/T, rs11893432 G/C, rs11889341 T/C, rs7574865 T/G and rs897200 C/T). Finally, we evaluated the association between STAT4 SNPs and T2D susceptibility. Our study may provide supplementary data for T2D risk assessment of specific population, and may also provide valuable reference for T2D individualized prevention.

\section{Methods}

\section{Study objects and sample collection}

After we fully obtained the consent of all participants, a total of 1001 Chinese Han people participated in this study (500 T2D patients and 501 healthy individuals with age and gender matched). Based on the genotyping results of all participants, we mainly used GCTA software (GCTA 1.26.0) to perform principal component analysis (PCA) and construct a kinship matrix to evaluate the genetic relationship between participants in this study [20]. The specific operations are as follows: (1) Plink software (PLINK v1.90b6.12) was used to convert the file format of genotyping data, which is necessary for PCA construction through GCTA software. When performing PCA, we set pca $=4$. Then we used $\mathrm{R}$ software (R4.0.3) to draw a scatter plot based on the file generated by GCTA. Finally, the genetic relationship between the participants was estimated according to the scatter plot. (2) We used the Plink software to convert the file format of the genotyping data. The GCTA software was used to calculate the genetic relationship matrix (GRM). Finally, the kinship matrix heat map was drawn using $\mathrm{R}$ software, and the kinship relationship between participants was estimated according to the kinship coefficient.

\section{Case group}

The 500 diabetic patients come from the First Affiliated Hospital of Xi'an Jiaotong university. Among them, 142 female, accounting for $28.4 \%$; 358 male, accounting for 71.6\%. T2D inclusion criteria are as follows: (1) outpatients or inpatients of the First Affiliated Hospital of Xi'an Jiaotong University; (2) patients who have been clearly diagnosed as T2D or newly diagnosed patients with T2D (diagnostic criteria: fasting blood glucose $\geq 7.0 \mathrm{mmol} / \mathrm{L} /$ OGTT $2 \mathrm{~h}$ blood glucose $\geq 11.1 \mathrm{mmol} / \mathrm{L} / \mathrm{random}$ blood glucose $\geq 11.1 \mathrm{mmol} / \mathrm{L}$ ); (3) the $\mathrm{T} 2 \mathrm{D}$ patients have no history of major mental trauma, and no history of genetic diseases: such as history of malignant tumors. All research subjects gave informed consents.

\section{Control group}

The 501 controls were healthy individuals selected at the same time and place as the above case group. Among them, 143 were female, accounting for 28.5\%; 358 were male, accounting for $71.5 \%$. The controls were selected according to the following requirements: (1) healthy individuals undergoing physical examination in the same hospital outpatient department at the same time; (2) fasting venous plasma glucose value $\leq 6.1 \mathrm{mmol} / \mathrm{L}$; (3) healthy individuals without complicated chronic diseases and surgical diseases, and tumor patients or people with tumor history are excluded; (4) the basic information (age and gender) of healthy individuals is not significantly different from the case group (excluding the difference in the distribution of exposure factors between case/control caused by confounding factors).

This study was conducted under the standard approved by the First Affiliated Hospital of Xi'an Jiaotong University. All participants took part in a questionnaire about demographic and anthropological information, such as: 
gender, height, weight, smoking, drinking, systolic blood pressure (SBP), diastolic blood pressure (DBP), and family history of diabetes etc.

\section{Sample collection}

We used vacuum blood collection tubes containing ethylenediaminetetraacetic acid (EDTA) to collect the fasting venous blood about $2 \mathrm{ml}$ of all participants in the morning, then placed it in a refrigerator at $-20^{\circ} \mathrm{C}$ to be stored until use.

\section{DNA extraction}

The whole genome DNA purification kit (GoldMag Co. Ltd. Xi'an, China) was used for this study, the specific experimental steps were shown in Additional file 1. The DNA was stored in the refrigerator at $-80^{\circ} \mathrm{C}$ until use.

\section{Selection of SNPS}

The selection of SNPs should follow the principle that the allele frequency of this locus is $\geq 5 \%$ in the study population. We also calculated the successful genotyping rate (call rate) of each candidate SNPs, then filtered out the SNPs with call rate $<90 \%$. Eliminating low-quality loci will help improve the reliability of the analysis results and reduce the false positive rate. According to the relevant literature and the data of STAT4 gene polymorphism in the database, we finally selected 5 sites of STAT4 gene for research (rs3821236 A/T, rs11893432 G/C, rs11889341 $\mathrm{T} / \mathrm{C}$, rs7574865 T/G and rs897200 C/T).

\section{Genotyping}

We use MassARRAY Assay Design software for primer design. And weused the MassARRAY system (Agena, San Diego, CA, USA) to genotype all SNPs.The MassARRAY platform is based on the MALDI-TOF (MatrixAssisted Laser Desorption/Ionization-Time of Flight) mass spectrometer, which has the characteristics of high throughput and cost-effectiveness. The iPLEX chemical method was used to generate SNP genotypes. The specific experimental steps are as follows: (1) The region targeted by multiplex analysis is amplified by PCR (catalog number 10500). (2) The PCR product is treated with shrimp alkaline phosphatase (SAP) to neutralize unincorporated nucleotides (Cat. No. \#08040). (3) Then perform an extension reaction to extend the PCR fragment by one base to the SNP site (catalog number 10136). (4) Then use MALDI-TOF to measure the quality of the obtained extension fragments to obtain the spectra of different mass peaks used for multiple reactions. Eventually we will successfully complete the genotyping.

\section{Quality control}

In order to verify the repeatability of the experiment, $10 \%$ of the DNA samples were randomly selected for repeated testing, and the agreement rate of the experimental results was $>99 \%$.

\section{Statistical analyses}

In this study, SPSS 17.0 statistical packages [21] was used to detect whether the SNPs of STAT4 conformed to Hardy-Weinberg equilibrium (HWE). After testing whether all candidate SNPs meet Hardy-Weinberg balance, the differences in the demographic characteristics (such as: age, gender, whether smoking, drinking, and $\mathrm{BMI})$ of participants in this study were tested by the chisquare test/t-test ( $t$ test was used for continuous variables such as age, and whether the mean value has statistical difference between the case group and the control group; the chi-square test was used for categorical variables such as gender, and whether the frequency distribution was statistically different between the case group and the control group). The $p$ value represents whether the result is statistically significant. The logistic regression model (Adjusted by gender and age) was used to analyze and calculate the odds ratio (OR) and 95\% confidence interval (CI) to evaluate the association between STAT4 polymorphism and type 2 diabetes risk. The reason why the logistic regression analysis only adjusted by age and gender is because the age and gender data of all participants are complete (There were large missing data on 'BMI, drinking, smoking'), which will effectively remove the influence of confounding factors on the accuracy of the results. The value of OR represents the odds ratio. When OR 1, it means that the factor has no effect on the occurrence of the disease; when OR $>1$, it is a risk factor; when $\mathrm{OR}<1$, it is a protective factor.

Using wild-type alleles as reference, SNPstats online tool software was used to estimate multiple genetic models (codominant, dominant, recessive, and log-additive models). We used multifactor dimensionality reduction (MDR) to assess 'SNP-SNP' interaction in diabetes risk. I We used one-way analysis of variance to assess the differences in clinical indicators between different genotypes (SPSS 17.0 statistical packages). All tests are two-sided tests, and $p<0.05$ is considered statistically significant.

\section{Result}

\section{Sample introduction and collection}

A total of 1,001 unrelated Chinese Han people participated in this study. We chose the 'case-control' experiment design type. The case group included 500 diabetic patients with an average age of $59.87 \pm 12.87$ years, the control group included 501 healthy individuals with an 
average age of $59.85 \pm 9.34$ years. It can be seen that there was no statistical difference in gender and age between the case group and the control group (Table 1). In addition, there was no statistical difference in smoking history or BMI between the control group and the case group, but the p-values were both closed to 0.05 . And there was a very significant difference in drinking history.

Table 1 The demographic and clinical characteristics of diabetic patients and controls

\begin{tabular}{|c|c|c|c|}
\hline Characteristics & $\begin{array}{l}\text { Controls } \\
(n=501)\end{array}$ & $\begin{array}{l}\text { Cases } \\
(n=500)\end{array}$ & $p$ value \\
\hline Age, years (mean $\pm S D$ ) & $59.85 \pm 9.34$ & $59.87 \pm 12.87$ & 0.973 \\
\hline$>60$ years old & $268(53 \%)$ & $240(48 \%)$ & \\
\hline$\leq 60$ years old & $233(47 \%)$ & $260(52 \%)$ & \\
\hline Gender & & & 0.960 \\
\hline Male & $358(71 \%)$ & $358(72 \%)$ & \\
\hline Female & 143(29\%) & $142(28 \%)$ & \\
\hline Drinking & & & $<0.001$ \\
\hline Yes & $103(21 \%)$ & $109(22 \%)$ & \\
\hline No & $140(28 \%)$ & $385(77 \%)$ & \\
\hline Smoking & & & 0.085 \\
\hline Yes & $98(20 \%)$ & $219(44 \%)$ & \\
\hline No & 164 (33\%) & $280(56 \%)$ & \\
\hline$B M I$ & & & 0.062 \\
\hline$>24$ & $130(26 \%)$ & $239(48 \%)$ & \\
\hline$\leq 24$ & $188(38 \%)$ & 203 (41\%) & \\
\hline FPG (mmol/L) & & & $<0.001$ \\
\hline Mean $\pm S D$ & $6.05 \pm 1.60$ & $7.35 \pm 3.40$ & \\
\hline Number & $386(77 \%)$ & 455 (91\%) & \\
\hline Creatinine (mg/dL) & & & 0.371 \\
\hline Mean \pm SD & $68.74 \pm 12.87$ & $71.20 \pm 52.66$ & \\
\hline Number & $385(77 \%)$ & 485 (97\%) & \\
\hline$A L T(I U / L)$ & & & 0.133 \\
\hline Mean $\pm S D$ & $27.66 \pm 31.35$ & $24.75 \pm 25.87$ & \\
\hline Number & $385(77 \%)$ & $492(98 \%)$ & \\
\hline TBA & & & 0.299 \\
\hline Mean $\pm S D$ & $6.66 \pm 18.22$ & $5.70 \pm 5.29$ & \\
\hline Number & $385(77 \%)$ & $421(84 \%)$ & \\
\hline Urea & & & $<0.001$ \\
\hline Mean $\pm S D$ & $5.42 \pm 2.78$ & $6.52 \pm 3.26$ & \\
\hline Number & 384 (77\%) & 484 (97\%) & \\
\hline$T C$ & & & $<0.001$ \\
\hline Mean $\pm S D$ & $4.93 \pm 4.00$ & $4.18 \pm 2.01$ & \\
\hline Number & 385 (77\%) & 496 (99\%) & \\
\hline
\end{tabular}

SD: standard deviation;

BMI: body mass index;

FPG: fasting plasma glucose;

ALT: alanine transaminase;

TBA: total bile acids;

TC: total cholesterol
Contributing to the above results might be the lack of sample data. The results of principal component analysis (Additional file 1: Fig. 1) and the kinship matrix heat map (Additional file 2: Fig. 2) can be seen that all participants can be considered to have no genetic relationship. The fasting blood glucose and urea content in the diabetes case group was higher than that in the control group, while the total cholesterol content was lower in the case group than in the control group. And the above indicators showed a significant difference between the two groups $(p<0.001)$, with statistical significance. The specific data information is summarized in Table 1.

\section{Association between STAT4 polymorphism and type 2 diabetes risk}

In this study, a total of 5 SNPs (rs3821236, rs11893432, rs11889341, rs7574865 and rs897200) were successfully genotyped. The call rate of all loci was more than 90\% (Table 2), which will help to improve the reliability of the results. The detailed information of candidate SNPs is listed in Table 2. All candidate SNPs are in HWE $(p>0.05)$. And the minor allele frequency (MAF) of all candidate SNPs are greater than $5 \%$ in the test population. The analysis results of HaploReg show that 5 SNPs are regulated by various factors, such as promoter histone marks, enhancer histone marks, motifs changed, NHGRI/EBI GWAS hits, GRASP QTL hits, Selected eQTL hits, etc. This study used logistic regression (Adjusted by gender and age) to test the association between SNPs and diabetes risk under different genetic models.

\section{Overall analysis}

Comprehensive analysis of all data, the result showed (Table 3) that among the 5 candidate SNPs in this study, only the rs3821236 polymorphism was associated with T2D risk, and the remaining four were not been found to be significantly associated with T2D risk $(p>0.05)$. Specifically, the results of this study showed that the genotype frequencies of rs3821236 (AA, AG and GG) in the case group were $22.6 \%, 50.6 \%$, and $26.8 \%$, while in the control group were $18.6 \%, 48.3 \%$, and $33.1 \%$, respectively. Among them, the allele (A vs. G, OR 1.23, CI 1.03-1.47, $p=0.020$ ) and homozygous (AA vs. GG, OR 1.51, CI $1.05-2.15, p=0.025)$ models were positively associated with increased risk of T2D. At the same time, we found that the rs3821236 polymorphism had a significant association with the increased risk of diabetes under dominant (GG vs. AA-AG, OR 1.36, CI 1.03-1.78, $p=0.029$ ) and log-additive models (OR 1.23, CI 1.03-1.47, $p=0.020)$. 


\section{Age and gender (Table 4)}

The study population was grouped according to age (60 years old as the dividing line) and gender (male and female) to analyze the association between genetic polymorphisms and T2Drisk in different subgroups. The rs3821236, rs11893432 and rs11889341 polymorphisms were positively associated with increased risk of T2D among participants aged $\leq 60$ years. Specifically, rs3821236 polymorphism was associated with an increased risk of T2D in allele (A vs. G, OR 1.45, CI $1.13-1.87, p=0.004$ ), homozygous (AA vs. GG, OR 2.16, CI 1.28-3.64, $p=0.004$ ), dominant (GG vs. AA-AG, OR 1.58, CI 1.06-2.35, $p=0.025$ ), recessive (AG-GG vs. AA, OR 1.75, CI 1.12-2.73, $p=0.014$ ), and logadditive models(OR 1.46, CI 1.13-1.90, $p=0.004$ ). We also found that rs 11893432 was positively associated with the risk of T2Din allele (C vs. G, OR 1.39, CI $1.08-1.79, p=0.010$ ), homozygous (CC vs. GG, OR 1.97, CI 1.17-3.32, $p=0.010$ ), dominant (CC vs. GG-GC, OR 1.54, CI 1.03-2.31, $p=0.036$ ), recessive (GC-CC vs. GG, OR 1.59, CI 1.03-2.46, $p=0.038)$ and log-additive models (OR 1.40, CI 1.08-1.82, $p=0.010$ ). However, we only observed an association between rs11889341 polymorphism and the increased risk of T2Din dominant (CC vs. TT-TC, OR 1.48, CI 1.03-2.13, $p=0.037$ ) and log-additive models (OR 1.34, CI 1.01-1.78, $p=0.046$ ). Conversely, among the participants over 60 years old, there was no association between the five candidate SNPs and the T2D risk. When the study population was divided by gender to analyze, the result showed that only rs11893432 was associated with the increased risk of T2D risk among female participants: rs11893432 was a risk factor for T2D in the allele (C vs. G, OR 1.44, CI $1.04-2.01, p=0.029$ ), homozygous (CC vs. GG, OR 2.19, CI 1.10-4.37, $p=0.026$ ) and log-additive models (OR 1.47, CI 1.05-2.07, $p=0.027$ ).

\section{BMI (Table 5)}

The subjects were grouped according to "body mass index' to analyze the association between candidate SNPs and T2D risk. The results showed that STAT4 rs11889341 (Dominant: OR 1.63, $p=0.035$ ) and rs7574865 (Heterozygote: OR 1.75, $p=0.021$; Dominant: OR 1.65, $p=0.030$ ) significantly increased T2D risk in participants with $\mathrm{BMI} \leq 24$. In participants with BMI $>24$, we did not find any evidence associated with T2D risk. In spite of this, the T2D risk of participants with BMI > 24 in our study almost all showed an increasing trend.

\section{Smoking and drinking (Table 6)}

The results showed that when the participants were grouped according to smoking status (Yes/No) for association analysis, we did not find any statistically significant results. Except for rs897200, the STAT4 rs3821236 (Allele: OR 1.42, $p=0.014$; Homozygote: OR 1.42, $p=0.014$; Heterozygote: OR 1.42, $p=0.014$; Dominant: OR 1.42, $p=0.014$; Log-additive: OR 1.42, $p=0.014$ ), rs11893432 (Allele: OR 1.43, $p=0.011$; Homozygote: OR 2.03, $p=0.015$; Dominant: OR 1.65, $p=0.019 ;$ Log-additive: OR 1.44, $p=0.012)$, rs11889341 (Heterozygote: OR 1.61, $p=0.025$; Dominant: OR 1.49, $p=0.036$ ) and rs7574865 (Heterozygote: OR 1.62, $p=0.023$ ) were all significantly associated with T2D risk among non-drinking participants. Although the five candidate SNPs had no potential association with the T2D risk among drinking participants, the T2D risk among drinking participants showed an increasing trend.

\section{T2D complications (Table 7)}

Finally, we grouped the case group according to whether they complicated with nephropathy or coronary heart disease (CHD) to evaluate the association between candidate SNPs and the risk of T2D complications. The results showed that (Table 7) only rs3821236 was potentially associated with the susceptibility to T2D complicated with nephropathy under heterozygous $(p=0.024)$ and dominant $(p=0.037)$ genetic models. At the same time, the results showed that the 5 candidate SNPs didn't associated with the susceptibility of T2D complicated with CHD.

\section{Differences in clinical indicators under different genotypes}

Finally, we also conducted an association study between the five candidate SNPs and clinical indicators s of T2D patients. The results showed (Table 8) that the level of clinical indicators associated with the candidate SNPs in this study were cystatin C, lysophosphatidic acid, and thyroxine. Specifically, the STAT4 rs11893432 was associated with LPa $(p=0.021)$; rs7574865 was associated with CysC $(p=0.033)$; while rs897200 had been found that was associated with T4 $(p=0.010)$. And the above data are statistically significant.

\section{MDR analysis}

Subsequently, we used MDR analysis to evaluate the SNP-SNP interaction. The interaction between these SNPs are described as Fig. 1. The blue line indicates that these 5 SNPs may have a redundancy effect in regulating the risk of diabetes. The specific information is summarized in Table 9. The results show that the best single-site 


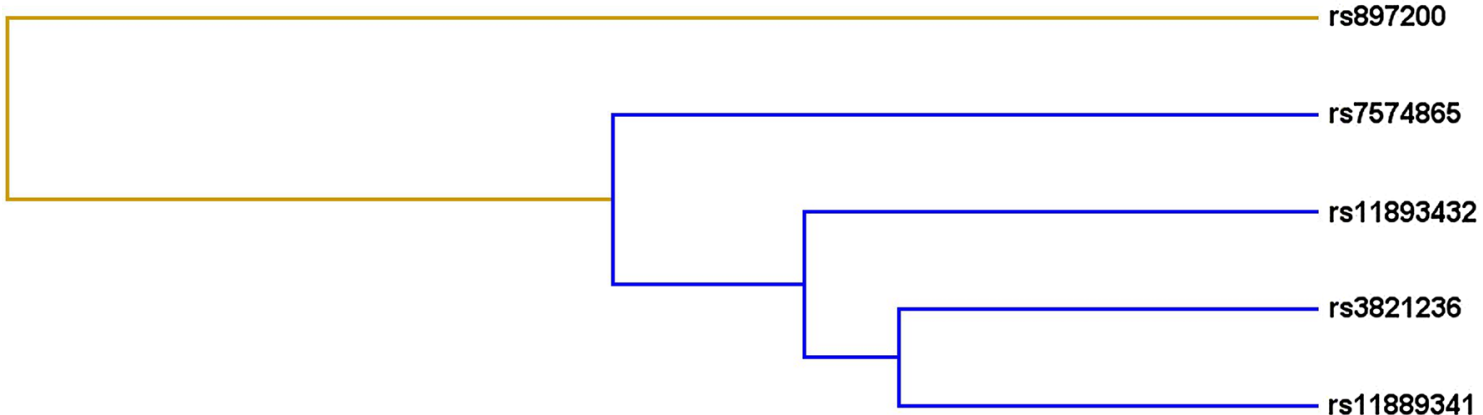

Fig. 1 Multifactor dimensionality reduction (MDR) analysis of STAT4 rs3821236, rs1 1893432, rs1 1889341, rs7574865 and rs897200 interaction. The colors in the tree diagram represent synergy (yellow) or redundancy (blue)

Table 2 The basic information of STAT4 polymorphisms

\begin{tabular}{|c|c|c|c|c|c|c|c|c|c|}
\hline \multirow[t]{2}{*}{ Gene } & \multirow[t]{2}{*}{ SNP ID } & \multirow[t]{2}{*}{ Chr:Position } & \multirow{2}{*}{$\begin{array}{l}\text { Alleles } \\
\text { (A/B) }\end{array}$} & \multirow[t]{2}{*}{ Call rate } & \multicolumn{2}{|l|}{ MAF } & \multirow{2}{*}{$\begin{array}{l}\text { HWE } \\
\text { ( } p \text { Value) }\end{array}$} & \multirow[t]{2}{*}{ Haploreg 4.1} & \multirow{2}{*}{$\begin{array}{l}\text { SNPinfo } \\
\text { web } \\
\text { serve }\end{array}$} \\
\hline & & & & & Cases & Controls & & & \\
\hline STAT4 & rs3821236 & Chr2: 191902758 & $\mathrm{~A} / \mathrm{G}$ & $100 \%$ & 0.479 & 0.427 & 0.784 & $\begin{array}{l}\text { Promoter histone marks; Enhancer histone } \\
\text { marks; Motifs changed; NHGRI/EBI GWAS hits; } \\
\text { GRASP QTL hits; Selected eQTL hits }\end{array}$ & \\
\hline STAT4 & rs11893432 & Chr2: 191921874 & $\mathrm{G} / \mathrm{C}$ & $100 \%$ & 0.483 & 0.441 & 0.928 & $\begin{array}{l}\text { Enhancer histone marks; Motifs changed; } \\
\text { Selected eQTL hits }\end{array}$ & \\
\hline STAT4 & rs11889341 & Chr2: 191943742 & $\mathrm{~T} / \mathrm{C}$ & $99.3 \%$ & 0.355 & 0.330 & 0.545 & $\begin{array}{l}\text { Promoter histone marks; Enhancer histone } \\
\text { marks; Motifs changed; NHGRI/EBI GWAS hits; }\end{array}$ & \\
\hline STAT4 & rs7574865 & Chr2: 191964633 & $\mathrm{~T} / \mathrm{G}$ & $100 \%$ & 0.347 & 0.327 & 0.223 & $\begin{array}{l}\text { Enhancer histone marks; Motifs changed; } \\
\text { NHGRI/EBI GWAS hits; GRASP QTL hits; }\end{array}$ & \\
\hline STAT4 & rs897200 & Chr2: 192017771 & $\mathrm{C} / \mathrm{T}$ & $100 \%$ & 0.489 & 0.494 & 0.475 & $\begin{array}{l}\text { Enhancer histone marks; DNAse; Proteins } \\
\text { bound; Motifs changed; NHGRI/EBI GWAS hits; } \\
\text { Selected eQTL hits }\end{array}$ & TFBS \\
\hline
\end{tabular}

HWE Hardy-Weinberg equilibrium, SNP single nucleotide polymorphisms, MAF minor allele frequency

$p>0.05$ indicates that the genotypes were in Hard-Weinberg Equilibrium;

model for predicting the risk of diabetes is: rs3821236 (testing accuracy $=0.515, \quad C V C=9 / 10, p=0.032$ ); the two-site model is: rs3821236, rs897200 (testing accuracy $=0.523, \mathrm{CVC}=5 / 10, p=0.011$ ); the three-site model is: rs3821236, rs11889341 and rs897200 (testing accuracy $=0.499, \mathrm{CVC}=5 / 10, \mathrm{p}=0.001)$; the four-site model is: rs3821236, rs11893432, rs11889341 and rs897200 (testing accuracy $=0.496, \mathrm{CVC}=8 / 10, p<0.0001$ ); the five-site models are: rs3821236, rs11893432, rs11889341, rs7574865 and rs897200 (testing accuracy $=0.501$, $\mathrm{CVC}=10 / 10, p<0.0001)$.

\section{Discussion}

Type 2 diabetes is the result of the interaction of genetic and environmental factors. In recent years, the association between genetic polymorphisms and diseases has been the focus of attention. Studies have found that STAT4 mainly induces Th1 response and inhibits Th2 response $[7,8]$. It plays an important role in the regulation of Th1/Th2 differentiation and the autoimmune diseases caused by this disorder. Multiple studies have shown that Th1/Th2 cytokine imbalance exists in T2D patients [10-12]. However, the specific mechanism of STAT4 in T2D is still unclear. Therefore, our study conducted a study on the association between STAT4 genetic polymorphisms and T2D risk in Chinese Han population. This study will supplement the data of T2D susceptibility-associated genetic loci.

Our results showed that only rs 3821236 was associated with type 2 diabetes risk among the five candidate SNPs of STAT4 (rs3821236 A/G, rs11893432 G/C, rs11889341 $\mathrm{T} / \mathrm{C}$, rs7574865 T/G and rs897200 $\mathrm{C} / \mathrm{T})$. STAT4 is an important transcriptional activator. After activation, it crosses the nuclear membrane into the nucleus in the form of a homodimer, and then initiates the transcription and expression of downstream target genes [22]. Numerous studies have found that the STAT4 rs3821236 genetic polymorphism is associated with multiple disease risks, such as systemic lupus erythematosus (SLE) [23], Systemic sclerosis [24] and juvenile idiopathic arthritis [25]. 
Table 3 Analysis of the association between diabetes risk and single nucleotide polymorphism of STAT4

\begin{tabular}{|c|c|c|c|c|c|c|}
\hline \multirow[t]{2}{*}{ SNP ID } & \multirow[t]{2}{*}{ Model } & \multirow[t]{2}{*}{ Genotype } & \multirow[t]{2}{*}{ Case } & \multirow[t]{2}{*}{ Control } & \multicolumn{2}{|c|}{ Adjusted by age and gender } \\
\hline & & & & & OR $(95 \% \mathrm{Cl})$ & $p$ \\
\hline \multirow[t]{10}{*}{ rs3821236 } & Allele & G & 521 & 574 & 1.00 & \\
\hline & & A & 479 & 428 & $1.23(1.03-1.47)$ & $0.020^{*}$ \\
\hline & Genotype & AA & 113 & 93 & $1.51(1.05-2.15)$ & $0.025^{*}$ \\
\hline & & $\mathrm{AG}$ & 253 & 242 & $1.30(0.97-1.73)$ & 0.077 \\
\hline & & GG & 134 & 166 & 1.00 & \\
\hline & Dominant & $A A-A G$ & 366 & 335 & $1.36(1.03-1.78)$ & $0.029^{*}$ \\
\hline & & GG & 134 & 166 & 1.00 & \\
\hline & Recessive & AA & 113 & 93 & $1.28(0.94-1.74)$ & 0.114 \\
\hline & & AG-GG & 387 & 408 & 1.00 & \\
\hline & Log-additive & - & - & - & $1.23(1.03-1.47)$ & $0.020^{*}$ \\
\hline \multirow[t]{10}{*}{ rs11893432 } & Allele & C & 517 & 560 & 1.00 & \\
\hline & & G & 483 & 442 & $1.18(0.99-1.41)$ & 0.060 \\
\hline & Genotype & GG & 116 & 98 & $1.40(0.98-1.99)$ & 0.064 \\
\hline & & GC & 251 & 246 & $1.21(0.90-1.61)$ & 0.208 \\
\hline & & $\mathrm{CC}$ & 133 & 157 & 1.00 & \\
\hline & Dominant & GG-GC & 367 & 344 & $1.26(0.96-1.66)$ & 0.098 \\
\hline & & CC & 133 & 157 & 1.00 & \\
\hline & Recessive & GG & 116 & 98 & $1.24(0.92-1.68)$ & 0.161 \\
\hline & & GC-CC & 384 & 403 & 1.00 & \\
\hline & Log-additive & - & - & - & $1.18(0.99-1.41)$ & 0.060 \\
\hline \multirow[t]{10}{*}{ rs11889341 } & Allele & C & 645 & 669 & 1.00 & \\
\hline & & $T$ & 355 & 329 & $1.12(0.93-1.35)$ & 0.233 \\
\hline & Genotype & $\pi T$ & 53 & 51 & $1.16(0.75-1.78)$ & 0.499 \\
\hline & & $\mathrm{TC}$ & 249 & 227 & $1.22(0.94-1.59)$ & 0.131 \\
\hline & & CC & 198 & 221 & 1.00 & \\
\hline & Dominant & TT-TC & 302 & 278 & $1.21(0.94-1.56)$ & 0.134 \\
\hline & & CC & 198 & 221 & 1.00 & \\
\hline & Recessive & $\mathrm{TT}$ & 53 & 51 & $1.04(0.69-1.56)$ & 0.845 \\
\hline & & TC-CC & 447 & 448 & 1.00 & \\
\hline & Log-additive & - & - & - & $1.13(0.93-1.36)$ & 0.220 \\
\hline \multirow[t]{10}{*}{ rs7574865 } & Allele & G & 653 & 673 & 1.00 & \\
\hline & & $\mathrm{T}$ & 347 & 327 & $1.09(0.91-1.32)$ & 0.344 \\
\hline & Genotype & $\mathrm{TT}$ & 49 & 47 & $1.14(0.73-1.77)$ & 0.571 \\
\hline & & TG & 249 & 233 & $1.17(0.90-1.51)$ & 0.253 \\
\hline & & GG & 202 & 220 & 1.00 & \\
\hline & Dominant & TT-TG & 298 & 280 & $1.16(0.90-1.49)$ & 0.247 \\
\hline & & GG & 202 & 220 & 1.00 & \\
\hline & Recessive & $T T$ & 49 & 47 & $1.05(0.69-1.60)$ & 0.828 \\
\hline & & TG-GG & 451 & 453 & 1.00 & \\
\hline & Log-additive & - & - & - & $1.10(0.91-1.34)$ & 0.322 \\
\hline \multirow[t]{10}{*}{ rs897200 } & Allele & T & 511 & 507 & 1.00 & \\
\hline & & C & 489 & 495 & $0.98(0.82-1.17)$ & 0.823 \\
\hline & Genotype & $\mathrm{CC}$ & 125 & 118 & $0.97(0.68-1.37)$ & 0.846 \\
\hline & & $\mathrm{CT}$ & 239 & 259 & $0.84(0.62-1.14)$ & 0.260 \\
\hline & & TT & 136 & 124 & 1.00 & \\
\hline & Dominant & CC-CT & 364 & 377 & $0.88(0.66-1.17)$ & 0.377 \\
\hline & & TT & 136 & 124 & 1.00 & \\
\hline & Recessive & $\mathrm{CC}$ & 125 & 118 & $1.08(0.81-1.45)$ & 0.594 \\
\hline & & CT-TT & 375 & 383 & 1.00 & \\
\hline & Log-additive & - & - & - & $0.98(0.82-1.17)$ & 0.822 \\
\hline
\end{tabular}


Table 4 The SNPS of STAT4 associated with diabetes risk in the subgroup tests

\begin{tabular}{|c|c|c|c|c|c|c|c|c|c|c|}
\hline SNP ID & Model & genotype & Case & Control & OR $(95 \% \mathrm{Cl})$ & $p$ & Case & Control & OR $(95 \% \mathrm{Cl})$ & $p$ \\
\hline Age, years & & & $>60$ & & & & $\leq 60$ & & & \\
\hline \multirow[t]{10}{*}{ rs3821236 } & Allele & G & 212 & 232 & 1.00 & & 267 & 196 & 1.00 & \\
\hline & & A & 268 & 304 & $1.04(0.81-1.33)$ & 0.777 & 253 & 270 & $1.45(1.13-1.87)$ & 0.004 \\
\hline & Genotype & GG & 73 & 90 & 1.00 & & 61 & 76 & 1.00 & \\
\hline & & $A A$ & 45 & 54 & $0.91(0.54-1.54)$ & 0.729 & 68 & 39 & $2.16(1.28-3.64)$ & 0.004 \\
\hline & & $A G$ & 122 & 124 & $1.15(0.76-1.73)$ & 0.507 & 131 & 118 & $1.39(0.91-2.12)$ & 0.127 \\
\hline & Dominant & GG & 73 & 90 & 1.00 & & 61 & 76 & 1.00 & \\
\hline & & $A A-A G$ & 167 & 178 & $1.08(0.73-1.58)$ & 0.710 & 199 & 157 & $1.58(1.06-2.35)$ & 0.025 \\
\hline & Recessive & $A G-G G$ & 195 & 214 & 1.00 & & 192 & 194 & 1.00 & \\
\hline & & AA & 45 & 54 & $0.84(0.53-1.33)$ & 0.454 & 68 & 39 & $1.75(1.12-2.73)$ & 0.014 \\
\hline & Log-additive & - & - & - & $0.98(0.76-1.26)$ & 0.864 & - & - & $1.46(1.13-1.90)$ & 0.004 \\
\hline \multirow[t]{10}{*}{ rs11893432 } & Allele & $C$ & 214 & 239 & 1.00 & & 269 & 203 & 1.00 & \\
\hline & & G & 266 & 297 & $1.00(0.78-1.28)$ & 0.998 & 251 & 263 & $1.39(1.08-1.79)$ & 0.010 \\
\hline & Genotype & CC & 74 & 85 & 1.00 & & 59 & 72 & 1.00 & \\
\hline & & GG & 48 & 56 & $0.89(0.53-1.49)$ & 0.664 & 68 & 42 & $1.97(1.17-3.32)$ & 0.010 \\
\hline & & CG & 118 & 127 & $1.00(0.66-1.51)$ & 1.000 & 133 & 119 & $1.39(0.91-2.13)$ & 0.130 \\
\hline & Dominant & $\mathrm{CC}$ & 74 & 85 & 1.00 & & 59 & 72 & 1.00 & \\
\hline & & GG-GC & 166 & 183 & $0.97(0.66-1.43)$ & 0.866 & 201 & 161 & $1.54(1.03-2.31)$ & 0.036 \\
\hline & Recessive & GC-CC & 192 & 212 & 1.00 & & 192 & 191 & 1.00 & \\
\hline & & GG & 48 & 56 & $0.84(0.53-1.33)$ & 0.454 & 68 & 42 & $1.59(1.03-2.46)$ & 0.038 \\
\hline & Log-additive & - & - & - & $0.95(0.74-1.23)$ & 0.695 & - & - & $1.40(1.08-1.82)$ & 0.010 \\
\hline \multirow[t]{10}{*}{ rs11889341 } & Allele & C & 158 & 181 & 1.00 & & 197 & 148 & 1.00 & \\
\hline & & T & 322 & 355 & $0.96(0.74-1.25)$ & 0.774 & 323 & 314 & $1.29(0.99-1.69)$ & 0.055 \\
\hline & Genotype & CC & 107 & 119 & 1.00 & & 91 & 102 & 1.00 & \\
\hline & & TT & 25 & 32 & $0.87(0.47-1.60)$ & 0.644 & 28 & 19 & $1.60(0.83-3.06)$ & 0.161 \\
\hline & & TC & 108 & 117 & $1.01(0.69-1.48)$ & 0.944 & 141 & 110 & $1.46(1.00-2.13)$ & 0.052 \\
\hline & Dominant & CC & 107 & 119 & 1.00 & & 91 & 102 & 1.00 & \\
\hline & & TT-TC & 133 & 149 & $0.98(0.68-1.41)$ & 0.925 & 169 & 129 & $1.48(1.03-2.13)$ & 0.037 \\
\hline & Recessive & TC-CC & 215 & 236 & 1.00 & & 232 & 212 & 1.00 & \\
\hline & & TT & 25 & 32 & $0.86(0.48-1.54)$ & 0.611 & 28 & 19 & $1.29(0.70-2.39)$ & 0.416 \\
\hline & Log-additive & - & - & - & $0.96(0.73-1.26)$ & 0.757 & - & - & $1.34(1.01-1.78)$ & 0.046 \\
\hline \multirow[t]{10}{*}{ rs7574865 } & Allele & G & 154 & 173 & 1.00 & & 193 & 154 & 1.00 & \\
\hline & & $\mathrm{T}$ & 326 & 361 & $0.99(0.76-1.28)$ & 0.915 & 327 & 312 & $1.20(0.92-1.56)$ & 0.182 \\
\hline & Genotype & GG & 109 & 121 & 1.00 & & 93 & 99 & 1.00 & \\
\hline & & TT & 23 & 27 & $1.01(0.54-1.91)$ & 0.972 & 26 & 20 & $1.32(0.68-2.53)$ & 0.412 \\
\hline & & TG & 108 & 119 & $1.01(0.69-1.47)$ & 0.967 & 141 & 114 & $1.33(0.91-1.94)$ & 0.138 \\
\hline & Dominant & GG & 109 & 121 & 1.00 & & 93 & 99 & 1.00 & \\
\hline & & TT-TG & 131 & 146 & $1.01(0.70-1.45)$ & 0.963 & 167 & 134 & $1.33(0.92-1.91)$ & 0.127 \\
\hline & Recessive & TG-GG & 217 & 240 & 1.00 & & 234 & 213 & 1.00 & \\
\hline & & TT & 23 & 27 & $1.01(0.55-1.85)$ & 0.981 & 26 & 20 & $1.12(0.60-2.08)$ & 0.722 \\
\hline & Log-additive & - & - & - & $1.01(0.76-1.33)$ & 0.963 & - & - & $1.22(0.92-1.62)$ & 0.177 \\
\hline \multirow[t]{8}{*}{ rs897200 } & Allele & T & 236 & 265 & 1.00 & & 245 & 224 & 1.00 & \\
\hline & & C & 244 & 271 & $0.99(0.77-1.27)$ & 0.931 & 275 & 242 & $0.96(0.75-1.24)$ & 0.765 \\
\hline & Genotype & $\mathrm{TT}$ & 65 & 69 & 1.00 & & 75 & 58 & 1.00 & \\
\hline & & CC & 61 & 66 & $1.13(0.68-1.88)$ & 0.626 & 60 & 49 & $0.91(0.54-1.52)$ & 0.713 \\
\hline & & $\mathrm{CT}$ & 114 & 133 & $1.00(0.64-1.54)$ & 0.990 & 125 & 126 & $0.75(0.49-1.15)$ & 0.191 \\
\hline & Dominant & TT & 65 & 69 & 1.00 & & 75 & 58 & 1.00 & \\
\hline & & $\mathrm{CC}-\mathrm{CT}$ & 175 & 199 & $1.04(0.69-1.57)$ & 0.847 & 185 & 175 & $0.80(0.53-1.19)$ & 0.268 \\
\hline & Recessive & CT-TT & 179 & 202 & 1.00 & & 200 & 184 & 1.00 & \\
\hline
\end{tabular}


Table 4 (continued)

\begin{tabular}{|c|c|c|c|c|c|c|c|c|c|c|}
\hline SNP ID & Model & genotype & Case & Control & OR $(95 \% \mathrm{Cl})$ & $p$ & Case & Control & OR (95\% Cl) & $p$ \\
\hline & & CC & 61 & 66 & $1.14(0.75-1.72)$ & 0.549 & 60 & 49 & $1.10(0.71-1.69)$ & 0.677 \\
\hline & Log-additive & - & - & - & $1.06(0.83-1.37)$ & 0.630 & - & - & $0.94(0.73-1.22)$ & 0.648 \\
\hline SNP ID & Model & genotype & Case & Control & OR $(95 \% \mathrm{Cl})$ & $p$ & Case & Control & OR $(95 \% \mathrm{Cl})$ & $p$ \\
\hline Gender & & & Male & & & & Female & & & \\
\hline \multirow[t]{10}{*}{ rs3821236 } & Allele & G & 336 & 302 & 1.00 & & 143 & 126 & 1.00 & \\
\hline & & $A$ & 380 & 414 & $1.21(0.98-1.49)$ & 0.071 & 141 & 160 & $1.29(0.93-1.79)$ & 0.154 \\
\hline & Genotype & GG & 101 & 122 & 1.00 & & 33 & 44 & 1.00 & \\
\hline & & AA & 79 & 66 & $1.45(0.95-2.20)$ & 0.085 & 34 & 27 & $1.69(0.85-3.33)$ & 0.132 \\
\hline & & $A G$ & 178 & 170 & $1.27(0.90-1.77)$ & 0.172 & 75 & 72 & $1.39(0.80-2.40)$ & 0.245 \\
\hline & Dominant & GG & 101 & 122 & 1.00 & & 33 & 44 & 1.00 & \\
\hline & & $A A-A G$ & 257 & 236 & $1.32(0.96-1.81)$ & 0.090 & 109 & 99 & $1.47(0.87-2.49)$ & 0.153 \\
\hline & Recessive & AG-GG & 279 & 292 & 1.00 & & 108 & 116 & 1.00 & \\
\hline & & AA & 79 & 66 & $1.25(0.87-1.81)$ & 0.227 & 34 & 27 & $1.36(0.77-2.40)$ & 0.295 \\
\hline & Log-additive & - & - & - & $1.21(0.98-1.49)$ & 0.073 & - & - & $1.30(0.93-1.83)$ & $0.125-$ \\
\hline \multirow[t]{10}{*}{ rs11893432 } & Allele & C & 338 & 322 & 1.00 & & 145 & 120 & 1.00 & \\
\hline & & G & 378 & 394 & $1.09(0.89-1.35)$ & 0.396 & 139 & 166 & $1.44(1.04-2.01)$ & 0.029 \\
\hline & Genotype & $\mathrm{CC}$ & 100 & 111 & 1.00 & & 33 & 46 & 1.00 & \\
\hline & & GG & 80 & 75 & $1.18(0.78-1.79)$ & 0.425 & 36 & 23 & $2.19(1.10-4.37)$ & 0.026 \\
\hline & & GC & 178 & 172 & $1.15(0.82-1.62)$ & 0.427 & 73 & 74 & $1.38(0.79-2.40)$ & 0.254 \\
\hline & Dominant & $\mathrm{CC}$ & 100 & 111 & 1.00 & & 33 & 46 & 1.00 & \\
\hline & & GG-GC & 258 & 247 & $1.16(0.84-1.60)$ & 0.367 & 109 & 97 & $1.57(0.93-2.66)$ & 0.092 \\
\hline & Recessive & GC-CC & 278 & 283 & 1.00 & & 106 & 120 & 1.00 & \\
\hline & & GG & 80 & 75 & $1.09(0.76-1.55)$ & 0.650 & 36 & 23 & $1.78(0.99-3.19)$ & 0.054 \\
\hline & Log-additive & - & - & - & $1.09(0.89-1.34)$ & 0.400 & - & - & $1.47(1.05-2.07)$ & 0.027 \\
\hline \multirow[t]{10}{*}{ rs11889341 } & Allele & C & 257 & 236 & 1.00 & & 98 & 93 & 1.00 & \\
\hline & & $\mathrm{T}$ & 459 & 476 & $1.13(0.91-1.41)$ & 0.275 & 186 & 193 & $1.09(0.77-1.55)$ & 0.615 \\
\hline & Genotype & $\mathrm{CC}$ & 140 & 157 & 1.00 & & 58 & 64 & 1.00 & \\
\hline & & $\mathrm{TT}$ & 39 & 37 & $1.18(0.71-1.96)$ & 0.516 & 14 & 14 & $1.11(0.49-2.52)$ & 0.810 \\
\hline & & $\mathrm{TC}$ & 179 & 162 & $1.24(0.91-1.69)$ & 0.178 & 70 & 65 & $1.19(0.73-1.94)$ & 0.489 \\
\hline & Dominant & $\mathrm{CC}$ & 140 & 157 & 1.00 & & 58 & 64 & 1.00 & \\
\hline & & TT-TC & 218 & 199 & $1.23(0.91-1.66)$ & 0.176 & 84 & 79 & $1.18(0.73-1.88)$ & 0.502 \\
\hline & Recessive & TC-CC & 319 & 319 & 1.00 & & 128 & 129 & 1.00 & \\
\hline & & $\mathrm{TT}$ & 39 & 37 & $1.06(0.66-1.70)$ & 0.827 & 14 & 14 & $1.01(0.46-2.21)$ & 0.981 \\
\hline & Log-additive & - & - & - & $1.14(0.91-1.43)$ & 0.261 & - & - & $1.10(0.77-1.58)$ & 0.599 \\
\hline \multirow[t]{10}{*}{ rs7574865 } & Allele & G & 252 & 233 & 1.00 & & 95 & 94 & 1.00 & \\
\hline & & $\mathrm{T}$ & 464 & 483 & $1.13(0.90-1.40)$ & 0.289 & 189 & 190 & $1.02(0.72-1.44)$ & 0.929 \\
\hline & Genotype & GG & 142 & 158 & 1.00 & & 60 & 62 & 1.00 & \\
\hline & & $\mathrm{TT}$ & 36 & 33 & $1.26(0.72-2.05)$ & 0.467 & 13 & 14 & $0.96(0.42-2.23)$ & 0.933 \\
\hline & & TG & 180 & 167 & $1.20(0.88-1.64)$ & 0.249 & 69 & 66 & $1.08(0.66-1.77)$ & 0.752 \\
\hline & Dominant & GG & 142 & 158 & 1.00 & & 60 & 62 & 1.00 & \\
\hline & & TT-TG & 216 & 200 & $1.20(0.89-1.62)$ & 0.225 & 82 & 80 & $1.06(0.66-1.70)$ & 0.802 \\
\hline & Recessive & TG-GG & 322 & 325 & 1.00 & & 13 & 14 & 1.00 & \\
\hline & & $\mathrm{TT}$ & 36 & 33 & $1.10(0.67-1.81)$ & 0.704 & 129 & 128 & $0.92(0.42-2.05)$ & 0.848 \\
\hline & Log-additive & - & - & - & $1.14(0.91-1.43)$ & 0.268 & - & - & $1.02(0.71-1.47)$ & 0.916 \\
\hline \multirow[t]{5}{*}{ rs897200 } & Allele & $\mathrm{T}$ & 346 & 350 & 1.00 & & 141 & 141 & 1.00 & \\
\hline & & $C$ & 370 & 366 & $0.98(0.79-1.20)$ & 0.833 & 143 & 145 & $1.01(0.73-1.41)$ & 0.934 \\
\hline & Genotype & $\mathrm{TT}$ & 102 & 91 & 1.00 & & 35 & 35 & 1.00 & \\
\hline & & $\mathrm{CC}$ & 90 & 83 & $0.97(0.64-1.46)$ & 0.875 & 34 & 33 & $1.03(0.53-2.01)$ & 0.930 \\
\hline & & $\mathrm{CT}$ & 166 & 184 & $0.80(0.57-1.14)$ & 0.227 & 73 & 75 & $0.97(0.55-1.72)$ & 0.927 \\
\hline
\end{tabular}


Table 4 (continued)

\begin{tabular}{lllllllllll}
\hline SNP ID & Model & genotype & Case & Control & OR $(\mathbf{9 5} \% \mathbf{C l})$ & $\boldsymbol{p}$ & Case & Control & OR (95\% CI) & $\boldsymbol{p}$ \\
\hline \multirow{2}{*}{ Dominant } & TT & 102 & 91 & 1.00 & & 35 & 35 & 1.00 \\
& CC-CT & 256 & 267 & $0.86(0.61-1.19)$ & 0.354 & 107 & 108 & $0.99(0.58-1.70)$ & 0.974 \\
& Recessive & CT-TT & 268 & 275 & 1.00 & & 108 & 110 & 1.00 & \\
& CC & 90 & 83 & $1.11(0.79-1.57)$ & 0.541 & 34 & 33 & $1.05(0.61-1.81)$ & 0.864 \\
& Log-additive & - & - & - & $0.98(0.80-1.20)$ & 0.833 & - & - & $1.02(0.73-1.42)$ & 0.933 \\
\hline
\end{tabular}

$\mathrm{Cl}$ confidence interval, $\mathrm{OR}$ odds ratio

$p$ : values were calculated by unconditional logistic regression analysis with adjustment for age and gender, ${ }^{*} p<0.05$ indicates statistical significance

etc. This study obtained similar results to previous studies: STAT4 rs3821236 was found to have a certain association with the risk of T2D in multiple genetic models (allele model, homozygous model, dominant model, etc.), whether we are performing an overall analysis or a stratified analysis.

In recent studies reported by Zhao et al. [10] and Mahlangu et al. [11], they all found that the differentiation regulation of Th1/Th2 played a certain role in T2D. And it has been found that STAT4 plays a certain role in the regulation of Th1/Th2 differentiation. Combined with the results of our study, we speculated that STAT4 rs3821236 may play a certain role in the differentiation and regulation of Th1/Th2, which may influence T2D susceptibility. However, this is only a speculation, which may need further study in larger sample size to confirm. Nevertheless, as far as we know, our study is the first to find evidence that STAT4 rs3821236 is potentially associated with the occurrence and development of T2D in Chinese Han population. It will provide new ideas for the individualized treatment or diagnosis of T2D.

On the other hand, genetic and environmental factors are interrelated in T2D and promote its development. The previous study has shown that age, obesity and unhealthy lifestyle are risk factors for T2D [26]. Therefore, this study also conducted a stratified analysis related to the above. Our results showed: among the population $\leq 60$ years old, rs3821236, rs11893432 and rs11889341 of STAT4 were significantly associated with increased T2D risk; among the population with $\mathrm{BMI}<24$, rs11889341 and rs7574865were significantly associated with increased risk of T2D; among the non-drinking population, rs3821236, rs11893432, rs11889341 and rs7574865 had a certain association with the increased risk ofT2D; in the analysis of whether the participants smoked, there was no significant association between STAT4 gene polymorphism and T2D risk. The above results seemed to be inconsistent with previous studies. We were pleasantly surprised to find that although there was no significant association between STAT4 gene polymorphism and T2D susceptibility among participants with potential T2D risk, it was showed an increasing trend of T2D risks among these participants. The result indicates that STAT4 gene polymorphism is associated with increased T2D risk, which may be greatly affected by genetic factors, while the environmental factors may have little effect.

In addition, we found that there are some differences between the results of our study and previous studies: STAT4 rs7574865 gene polymorphism is a risk factor for increasing the risk of diabetes in Asians and Caucasians [27], while according to the results of this study, rs7574865 was only associated with the clinical indicator (cystatin $C, p=0.033$ ). However, it is not sufficient to prove that rs7574865 is associated with T2D risk. We speculate that the causes for the above differences may be different research populations, inconsistent sample sizes and different research environments etc.

Our study provides data supplement for the study of the association between STAT4 gene polymorphism and the risk of T2D in Chinese Han population: there is a certain association between the two. However, this study still has certain limitations. Because of the small sample size and missing sample data (BMI, drinking, smoking). Only two baselines of age and gender were adjusted in the logistic regression to ensure the accuracy of the results. In subsequent studies, we need to further expand the sample size to continue the study, so as to more strongly confirm the results of our study.

\section{Conclusion}

In summary, the study is the first study of the association between STAT4 gene polymorphism and T2D risk in Chinese Han population. Our results suggest that STAT4 gene polymorphism (rs3821236, rs11893432, rs11889341, rs7574865, rs897200) has a potential association with the risk of T2D in the Chinese Han population. It provides supplementary data for the in-depth study of the association between the STAT4 gene and T2D risk. And it can provide a theoretical and scientific basis for the preliminary molecular basis of prevention and treatment for T2D from a genetic perspective. 
Table 5 The SNPS of STAT4 associated with T2D risk in the subgroup tests (BMI)

\begin{tabular}{|c|c|c|c|c|c|c|}
\hline SNP ID & Model & Genotype & $\begin{array}{l}\text { OR }(95 \% \mathrm{Cl}) \\
<24\end{array}$ & $p$ & $\begin{array}{l}\text { OR }(95 \% \mathrm{Cl}) \\
>24\end{array}$ & $p$ \\
\hline \multirow[t]{10}{*}{ rs3821236 } & Allele & A & $1.30(0.95-1.79)$ & 0.099 & $1.1(0.84-1.44)$ & 0.498 \\
\hline & & G & 1.00 & & 1.00 & \\
\hline & Genotype & AA & $1.82(0.94-3.53)$ & 0.076 & $1.18(0.69-2.04)$ & 0.544 \\
\hline & & AG & $1.35(0.81-2.24)$ & 0.250 & $1.17(0.73-1.87)$ & 0.505 \\
\hline & & GG & 1.00 & & 1.00 & \\
\hline & Dominant & AA-AG & $1.46(0.90-2.36)$ & 0.123 & $1.18(0.76-1.82)$ & 0.470 \\
\hline & & GG & 1.00 & & 1.00 & \\
\hline & Recessive & AA & $1.51(0.85-2.71)$ & 0.164 & $1.07(0.68-1.68)$ & 0.778 \\
\hline & & AG-GG & 1.00 & & 1.00 & \\
\hline & Log-additive & - & $1.35(0.98-1.87)$ & 0.070 & $1.09(0.83-1.43)$ & 0.538 \\
\hline \multirow[t]{10}{*}{ rs11893432 } & Allele & G & $1.34(0.98-1.84)$ & 0.066 & $1.03(0.79-1.35)$ & 0.830 \\
\hline & & C & 1.00 & & 1.00 & \\
\hline & Genotype & GG & $1.83(0.95-3.52)$ & 0.070 & $1.07(0.62-1.84)$ & 0.815 \\
\hline & & GC & $1.51(0.91-2.52)$ & 0.113 & $0.88(0.55-1.42)$ & 0.609 \\
\hline & & $C C$ & 1.00 & & 1.00 & \\
\hline & Dominant & GG-GC & $1.59(0.98-2.58)$ & 0.059 & $0.94(0.60-1.47)$ & 0.792 \\
\hline & & $\mathrm{CC}$ & 1.00 & & 1.00 & \\
\hline & Recessive & GG & $1.42(0.80-2.51)$ & 0.236 & $1.16(0.74-1.81)$ & 0.521 \\
\hline & & GC-CC & 1.00 & & 1.00 & \\
\hline & Log-additive & - & $1.37(0.99-1.90)$ & 0.055 & $1.03(0.79-1.36)$ & 0.818 \\
\hline \multirow[t]{10}{*}{ rs11889341 } & Allele & $\mathrm{T}$ & $1.41(1.01-1.98)$ & 0.115 & $1.86(0.65-2.14)$ & 0.282 \\
\hline & & C & 1.00 & & 1.00 & \\
\hline & Genotype & $\pi$ & $1.62(0.72-3.63)$ & 0.243 & $1.73(1.38-2.41)$ & 0.353 \\
\hline & & $\mathrm{TC}$ & $1.63(1.02-2.62)$ & 0.143 & $1.29(0.52-2.21)$ & 0.279 \\
\hline & & CC & 1.00 & & 1.00 & \\
\hline & Dominant & TT-TC & $1.63(1.04-2.56)$ & $0.035^{*}$ & $1.78(0.52-2.17)$ & 0.230 \\
\hline & & CC & 1.00 & & 1.00 & \\
\hline & Recessive & $\pi$ & $1.27(0.58-2.75)$ & 0.549 & $1.84(0.45-2.54)$ & 0.564 \\
\hline & & TC-CC & 1.00 & & 1.00 & \\
\hline & Log-additive & - & $1.41(0.99-2.00)$ & 0.059 & $1.84(0.62-2.33)$ & 0.239 \\
\hline \multirow[t]{10}{*}{ rs75748665 } & Allele & $\mathrm{T}$ & $1.36(0.97-1.91)$ & 0.073 & $1.37(0.66-2.16)$ & 0.342 \\
\hline & & G & 1.00 & & 1.00 & \\
\hline & Genotype & $\pi$ & $1.26(0.58-2.74)$ & 0.555 & $1.48(0.39-2.15)$ & 0.483 \\
\hline & & TG & $1.75(1.09-2.83)$ & $0.021^{*}$ & $1.80(0.53-2.21)$ & 0.288 \\
\hline & & GG & 1.00 & & 1.00 & \\
\hline & Dominant & TT-TG & $1.65(1.05-2.59)$ & $0.030^{*}$ & $1.28(0.53-1.99)$ & 0.263 \\
\hline & & GG & 1.00 & & 1.00 & \\
\hline & Recessive & $\pi$ & $0.96(0.46-2.03)$ & 0.921 & $1.19(0.47-1.89)$ & 0.721 \\
\hline & & TG-GG & 1.00 & & 1.00 & \\
\hline & Log-additive & - & $1.33(0.94-1.89)$ & 0.108 & $1.65(0.63-2.26)$ & 0.306 \\
\hline \multirow[t]{10}{*}{ rs897200 } & Allele & C & $0.83(0.60-1.13)$ & 0.229 & $1.16(0.89-1.52)$ & 0.274 \\
\hline & & $\mathrm{T}$ & 1.00 & & 1.00 & \\
\hline & Genotype & $\mathrm{CC}$ & $0.66(0.34-1.27)$ & 0.214 & $1.33(0.78-2.26)$ & 0.294 \\
\hline & & CT & $1.20(0.71-2.03)$ & 0.503 & $0.86(0.53-1.39)$ & 0.538 \\
\hline & & $\pi$ & 1.00 & & 1.00 & \\
\hline & Dominant & CC-CT & $1.02(0.62-1.68)$ & 0.944 & $1.02(0.65-1.59)$ & 0.933 \\
\hline & & $\pi$ & 1.00 & & 1.00 & \\
\hline & Recessive & $\mathrm{CC}$ & $0.59(0.34-1.02)$ & 0.059 & $1.46(0.95-2.25)$ & 0.085 \\
\hline & & CT-TT & 1.00 & & 1.00 & \\
\hline & Log-additive & - & $0.84(0.60-1.16)$ & 0.287 & $1.16(0.89-1.51)$ & 0.266 \\
\hline
\end{tabular}

$B M I$ body mass index, $C l$ confidence interval, $O R$ odds ratio 
Table 6 The SNPs of STAT4 associated with T2D risk in the subgroup tests (smoking and drinking status)

\begin{tabular}{|c|c|c|c|c|c|c|c|c|c|c|}
\hline \multirow[t]{3}{*}{ SNP ID } & \multirow[t]{3}{*}{ Model } & \multirow[t]{3}{*}{ Genotype } & \multicolumn{4}{|l|}{ Smoking } & \multicolumn{4}{|l|}{ Drinking } \\
\hline & & & OR $(95 \% \mathrm{Cl})$ & $p$ & OR $(95 \% \mathrm{Cl})$ & $p$ & OR $(95 \% \mathrm{Cl})$ & $p$ & & $p$ \\
\hline & & & Yes & & No & & Yes & & No & \\
\hline \multirow[t]{10}{*}{ rs3821236 } & Allele & A & $1.31(0.93-1.84)$ & 0.119 & $1.27(0.96-1.67)$ & 0.092 & $1.29(0.68-2.45)$ & 0.964 & $1.42(1.07-1.87)$ & $0.014^{*}$ \\
\hline & & G & 1.00 & & 1.00 & & 1.00 & & 1.00 & \\
\hline & Genotype & AA & $1.98(0.93-4.2)$ & 0.076 & $1.53(0.88-2.65)$ & 0.132 & $1.07(0.48-2.38)$ & 0.866 & $1.87(1.07-3.27)$ & $0.027^{*}$ \\
\hline & & $A G$ & $1.08(0.62-1.86)$ & 0.788 & $1.42(0.90-2.22)$ & 0.129 & $1.14(0.39-2.39)$ & 0.344 & $1.73(1.12-2.69)$ & $0.014^{*}$ \\
\hline & & GG & 1.00 & & 1.00 & & 1.00 & & 1.00 & \\
\hline & Dominant & $A A-A G$ & $1.25(0.74-2.11)$ & 0.396 & $1.45(0.95-2.21)$ & 0.084 & $1.02(0.45-1.89)$ & 0.510 & $1.77(1.17-2.68)$ & $0.006^{*}$ \\
\hline & & GG & 1.00 & & 1.00 & & 1.00 & & 1.00 & \\
\hline & Recessive & AA & $1.89(0.97-3.68)$ & 0.063 & $1.24(0.76-2.00)$ & 0.388 & $1.30(0.65-2.59)$ & 0.460 & $1.35(0.82-2.21)$ & 0.241 \\
\hline & & AG-GG & 1.00 & & 1.00 & & 1.00 & & 1.00 & \\
\hline & Log-additive & - & $1.34(0.94-1.91)$ & 0.102 & $1.25(0.95-1.65)$ & 0.108 & $1.00(0.67-1.48)$ & 0.990 & $1.42(1.07-1.88)$ & $0.015^{*}$ \\
\hline \multirow[t]{10}{*}{ rs11893432 } & Allele & G & $1.12(0.80-1.57)$ & 0.511 & $1.26(0.96-1.66)$ & 0.097 & $1.28(0.55-2.18)$ & 0.261 & $1.43(1.09-1.89)$ & $0.011^{*}$ \\
\hline & & c & 1.00 & & 1.00 & & 1.00 & & 1.00 & \\
\hline & Genotype & GG & $1.25(0.63-2.51)$ & 0.525 & $1.63(0.93-2.88)$ & 0.090 & $1.09(0.33-1.88)$ & 0.347 & $2.03(1.15-3.61)$ & $0.015^{*}$ \\
\hline & & GC & $1.08(0.62-1.90)$ & 0.777 & $1.16(0.74-1.83)$ & 0.510 & $1.16(0.32-2.13)$ & 0.114 & $1.52(0.98-2.37)$ & 0.062 \\
\hline & & CC & 1.00 & & 1.00 & & 1.00 & & 1.00 & \\
\hline & Dominant & GG-GC & $1.13(0.67-1.92)$ & 0.649 & $1.29(0.84-1.97)$ & 0.250 & $1.23(0.35-2.14)$ & 0.126 & $1.65(1.09-2.51)$ & $0.019^{*}$ \\
\hline & & CC & 1.00 & & 1.00 & & 1.00 & & 1.00 & \\
\hline & Recessive & GG & $1.19(0.66-2.16)$ & 0.568 & $1.48(0.91-2.42)$ & 0.115 & $1.05(0.50-1.92)$ & 0.882 & $1.56(0.94-2.59)$ & 0.086 \\
\hline & & GC-CC & 1.00 & & 1.00 & & 1.00 & & 1.00 & \\
\hline & Log-additive & - & $1.12(0.79-1.58)$ & 0.531 & $1.27(0.96-1.67)$ & 0.097 & $1.12(0.56-1.99)$ & 0.289 & $1.44(1.08-1.91)$ & $0.012^{*}$ \\
\hline \multirow[t]{10}{*}{ rs11889341 } & Allele & T & $1.17(0.82-1.67)$ & 0.383 & $1.09(0.81-1.45)$ & 0.581 & $0.98(0.65-1.46)$ & 0.906 & $1.22(0.91-1.64)$ & 0.184 \\
\hline & & C & 1.00 & & 1.00 & & 1.00 & & 1.00 & \\
\hline & Genotype & $\pi T$ & $1.37(0.56-3.36)$ & 0.486 & $1.13(0.59-2.18)$ & 0.713 & $1.27(0.49-3.31)$ & 0.621 & $1.09(0.57-2.08)$ & 0.802 \\
\hline & & TC & $1.24(0.75-2.06)$ & 0.403 & $1.18(0.78-1.79)$ & 0.429 & $1.07(0.43-1.97)$ & 0.371 & $1.61(1.06-2.44)$ & $0.025^{*}$ \\
\hline & & CC & 1.00 & & 1.00 & & 1.00 & & 1.00 & \\
\hline & Dominant & TT-TC & $1.26(0.77-2.06)$ & 0.354 & $1.17(0.79-1.74)$ & 0.430 & $1.22(0.48-2.16)$ & 0.534 & $1.49(1.01-2.21)$ & $0.036^{*}$ \\
\hline & & CC & 1.00 & & 1.00 & & 1.00 & & 1.00 & \\
\hline & Recessive & $\pi$ & $1.22(0.52-2.84)$ & 0.652 & $1.04(0.56-1.93)$ & 0.905 & $1.47(0.59-3.62)$ & 0.406 & $0.85(0.46-1.58)$ & 0.615 \\
\hline & & TC-CC & 1.00 & & 1.00 & & 1.00 & & 1.00 & \\
\hline & Log-additive & - & $1.20(0.82-1.77)$ & 0.354 & $1.10(0.82-1.48)$ & 0.519 & $1.08(0.65-1.89)$ & 0.935 & $1.22(0.9-1.65)$ & 0.202 \\
\hline \multirow[t]{10}{*}{ rs7574865 } & Allele & T & $1.21(0.84-1.73)$ & 0.303 & $1.03(0.77-1.38)$ & 0.832 & $1.06(0.64-2.23)$ & 0.835 & $1.18(0.88-1.58)$ & 0.272 \\
\hline & & G & 1.00 & & 1.00 & & 1.00 & & 1.00 & \\
\hline & Genotype & $\pi$ & $1.73(0.65-4.61)$ & 0.275 & $1.07(0.50-1.87)$ & 0.927 & $1.33(0.47-3.77)$ & 0.589 & $0.95(0.50-1.82)$ & 0.883 \\
\hline & & TG & $1.18(0.71-1.95)$ & 0.520 & $1.20(0.79-1.81)$ & 0.390 & $1.05(0.42-2.02)$ & 0.311 & $1.62(1.07-2.45)$ & $0.023^{*}$ \\
\hline & & GG & 1.00 & & 1.00 & & 1.00 & & 1.00 & \\
\hline & Dominant & TT-TG & $1.24(0.76-2.02)$ & 0.387 & $1.15(0.78-1.70)$ & 0.482 & $1.11(0.47-2.04)$ & 0.451 & $1.46(0.99-2.16)$ & 0.058 \\
\hline & & GG & 1.00 & & 1.00 & & 1.00 & & 1.00 & \\
\hline & Recessive & $\pi$ & $1.58(0.61-4.04)$ & 0.344 & $1.08(0.47-1.95)$ & 0.697 & $1.56(0.58-4.22)$ & 0.377 & $0.75(0.40-1.38)$ & 0.349 \\
\hline & & TG-GG & 1.00 & & 1.00 & & 1.00 & & 1.00 & \\
\hline & Log-additive & - & $1.25(0.84-1.85)$ & 0.267 & $1.05(0.78-1.42)$ & 0.728 & $0.96(0.62-1.48)$ & 0.843 & $1.17(0.86-1.58)$ & 0.314 \\
\hline \multirow[t]{10}{*}{ rs897200 } & Allele & c & $1.02(0.66-1.89)$ & 0.641 & $1.23(0.94-1.62)$ & 0.136 & $0.93(0.63-1.36)$ & 0.706 & $1.21(0.92-1.60)$ & 0.165 \\
\hline & & T & 1.00 & & 1.00 & & 1.00 & & 1.00 & \\
\hline & Genotype & CC & $1.04(0.43-2.02)$ & 0.600 & $1.51(0.86-2.65)$ & 0.153 & $1.38(0.43-2.03)$ & 0.737 & $1.62(0.90-2.91)$ & 0.111 \\
\hline & & CT & $1.06(0.43-1.86)$ & 0.358 & $1.20(0.76-1.90)$ & 0.440 & $1.13(0.59-2.16)$ & 0.711 & $0.88(0.56-1.39)$ & 0.578 \\
\hline & & $\pi$ & 1.00 & & 1.00 & & 1.00 & & 1.00 & \\
\hline & Dominant & $\mathrm{CC}-\mathrm{CT}$ & $1.19(0.46-2.35)$ & 0.388 & $1.28(0.83-1.98)$ & 0.261 & $1.03(0.57-1.87)$ & 0.924 & $1.04(0.67-1.62)$ & 0.849 \\
\hline & & $\pi$ & 1.00 & & 1.00 & & 1.00 & & 1.00 & \\
\hline & Recessive & CC & $1.00(0.57-1.92)$ & 0.973 & $1.34(0.83-2.16)$ & 0.230 & $1.22(0.44-1.91)$ & 0.525 & $1.76(1.06-2.91)$ & $0.028^{*}$ \\
\hline & & CT-TT & 1.00 & & 1.00 & & 1.00 & & 1.00 & \\
\hline & Log-additive & - & $1.01(0.66-2.06)$ & 0.582 & $1.23(0.93-1.62)$ & 0.154 & $0.94(0.66-1.36)$ & 0.750 & $1.23(0.93-1.62)$ & 0.149 \\
\hline
\end{tabular}


Table 6 (continued)

Cl confidence interval, $O R$ odds ratio

$p$ values were calculated by unconditional logistic regression analysis with adjustment for age and gender; ${ }^{*} p<0.05$ indicates statistical significance

Table 7 Correlation between STAT4 gene polymorphism and the occurrence of diabetes complications

\begin{tabular}{|c|c|c|c|c|c|c|c|c|c|c|}
\hline \multirow[t]{2}{*}{ SNP ID } & \multirow[t]{2}{*}{ Model } & \multirow[t]{2}{*}{ Genotype } & \multicolumn{4}{|c|}{ T2D complicated with nephropathy } & \multicolumn{4}{|c|}{ T2D complicated with CHD } \\
\hline & & & DN & No DN & OR $(95 \% \mathrm{Cl})$ & $p$ & Case & Control & OR $(95 \% \mathrm{Cl})$ & $p$ \\
\hline \multirow[t]{10}{*}{ rs3821236 } & Allele & A & 127 & 352 & $0.78(0.59-1.02)$ & 0.073 & 127 & 352 & 1.14(0.86-1.52) & 0.359 \\
\hline & & G & 165 & 356 & 1.00 & & 125 & 396 & 1.00 & \\
\hline & Genotype & $\mathrm{AA}$ & 30 & 83 & $0.65(0.37-1.14)$ & 0.131 & 30 & 83 & $1.41(0.77-2.60)$ & 0.266 \\
\hline & & $A G$ & 67 & 186 & $0.62(0.40-0.99)$ & $0.024^{*}$ & 67 & 186 & 1.35(0.81-2.25) & 0.251 \\
\hline & & GG & 49 & 85 & 1.00 & & 29 & 105 & 1.00 & \\
\hline & Dominant & $A A-A G$ & 97 & 269 & $0.63(0.41-0.97)$ & $0.037^{*}$ & 97 & 269 & $1.37(0.84-2.23)$ & 0.207 \\
\hline & & GG & 49 & 85 & 1.00 & & 29 & 105 & 1.00 & \\
\hline & Recessive & $\mathrm{AA}$ & 30 & 83 & $0.88(0.54-1.42)$ & 0.589 & 30 & 83 & $1.16(0.70-1.91)$ & 0.567 \\
\hline & & AG-GG & 116 & 271 & 1.00 & & 96 & 291 & 1.00 & \\
\hline & Log-additive & - & - & - & $0.79(0.59-1.05)$ & 0.099 & - & - & 1.19(0.88-1.61) & 0.252 \\
\hline \multirow[t]{10}{*}{ rs11893432 } & Allele & G & 131 & 352 & $0.82(0.63-1.08)$ & 0.163 & 126 & 357 & $1.10(0.82-1.46)$ & 0.532 \\
\hline & & C & 161 & 356 & 1.00 & & 126 & 391 & 1.00 & \\
\hline & Genotype & GG & 30 & 86 & $0.71(0.40-1.24)$ & 0.227 & 30 & 86 & $1.29(0.70-2.35)$ & 0.416 \\
\hline & & GC & 71 & 180 & $0.78(0.49-1.23)$ & 0.280 & 66 & 185 & $1.26(0.76-2.10)$ & 0.371 \\
\hline & & CC & 45 & 88 & 1.00 & & 30 & 103 & 1.00 & \\
\hline & Dominant & GG-GC & 101 & 266 & $0.75(0.49-1.17)$ & 0.203 & 96 & 271 & $1.27(0.78-2.06)$ & 0.334 \\
\hline & & CC & 45 & 88 & 1.00 & & 30 & 103 & 1.00 & \\
\hline & Recessive & GG & 30 & 86 & $0.83(0.51-1.35)$ & 0.455 & 30 & 86 & $1.10(0.67-1.81)$ & 0.704 \\
\hline & & GC-CC & 116 & 268 & 1.00 & & 96 & 288 & 1.00 & \\
\hline & Log-additive & - & - & - & $0.84(0.63-1.11)$ & 0.212 & - & - & $1.14(0.84-1.53)$ & 0.402 \\
\hline \multirow[t]{10}{*}{ rs1 1889341} & Allele & T & 97 & 258 & $0.87(0.65-1.16)$ & 0.333 & 81 & 274 & $0.82(0.61-1.11)$ & 0.198 \\
\hline & & C & 195 & 450 & 1.00 & & 171 & 474 & 1.00 & \\
\hline & Genotype & TT & 15 & 38 & $0.81(0.41-1.62)$ & 0.558 & 10 & 43 & $0.56(0.25-1.25)$ & 0.156 \\
\hline & & $\mathrm{TC}$ & 67 & 182 & $0.79(0.52-1.21)$ & 0.280 & 61 & 188 & $0.89(0.57-1.37)$ & 0.589 \\
\hline & & CC & 64 & 134 & 1.00 & & 55 & 143 & 1.00 & \\
\hline & Dominant & TT-TC & 82 & 220 & $0.80(0.54-1.19)$ & 0.268 & 71 & 231 & $0.82(0.54-1.26)$ & 0.370 \\
\hline & & CC & 64 & 134 & 1.00 & & 55 & 143 & 1.00 & \\
\hline & Recessive & $\mathrm{TT}$ & 15 & 38 & $0.92(0.48-1.77)$ & 0.807 & 10 & 43 & $0.60(0.28-1.28)$ & 0.188 \\
\hline & & TC-CC & 131 & 316 & 1.00 & & 116 & 331 & 1.00 & \\
\hline & Log-additive & - & - & - & $0.86(0.63-1.17)$ & 0.339 & - & - & $0.80(0.58-1.12)$ & 0.192 \\
\hline \multirow[t]{10}{*}{ rs7574865 } & Allele & T & 96 & 251 & $0.89(0.67-1.19)$ & 0.437 & 82 & 265 & $0.88(0.65-1.19)$ & 0.405 \\
\hline & & G & 196 & 457 & 1.00 & & 170 & 483 & 1.00 & \\
\hline & Genotype & $\mathrm{TT}$ & 14 & 35 & $0.90(0.45-1.83)$ & 0.775 & 9 & 40 & $0.65(0.29-1.47)$ & 0.302 \\
\hline & & TG & 68 & 181 & $0.84(0.55-1.27)$ & 0.410 & 64 & 185 & $1.04(0.67-1.61)$ & 0.869 \\
\hline & & GG & 64 & 138 & 1.00 & & 53 & 149 & 1.00 & \\
\hline & Dominant & TT-TG & 82 & 216 & $0.85(0.57-1.27)$ & 0.423 & 73 & 225 & $0.97(0.63-1.48)$ & 0.882 \\
\hline & & GG & 64 & 138 & 1.00 & & 53 & 149 & 1.00 & \\
\hline & Recessive & $\mathrm{TT}$ & 14 & 35 & $0.99(0.51-1.94)$ & 0.980 & 9 & 40 & $0.64(0.29-1.39)$ & 0.259 \\
\hline & & TG-GG & 132 & 319 & 1.00 & & 117 & 334 & 1.00 & \\
\hline & Log-additive & - & - & - & $0.91(0.66-1.24)$ & 0.530 & - & - & $0.90(0.65-1.25)$ & 0.524 \\
\hline \multirow[t]{4}{*}{ rs897200 } & Allele & C & 147 & 342 & $1.09(0.83-1.43)$ & 0.558 & 129 & 360 & $1.13(0.85-1.5)$ & 0.400 \\
\hline & & T & 145 & 366 & 1.00 & & 123 & 388 & 1.00 & \\
\hline & Genotype & CC & 35 & 90 & $1.10(0.63-1.93)$ & 0.741 & 35 & 90 & $1.16(0.65-2.07)$ & 0.605 \\
\hline & & CT & 77 & 162 & $1.44(0.89-2.33)$ & 0.139 & 59 & 180 & $1.05(0.63-1.75)$ & 0.852 \\
\hline
\end{tabular}


Table 7 (continued)

\begin{tabular}{|c|c|c|c|c|c|c|c|c|c|c|}
\hline \multirow[t]{2}{*}{ SNP ID } & \multirow[t]{2}{*}{ Model } & \multirow[t]{2}{*}{ Genotype } & \multicolumn{4}{|c|}{ T2D complicated with nephropathy } & \multicolumn{4}{|c|}{ T2D complicated with CHD } \\
\hline & & & DN & No DN & OR $(95 \% \mathrm{Cl})$ & $p$ & Case & Control & OR $(95 \% \mathrm{Cl})$ & $p$ \\
\hline & & $\mathrm{TT}$ & 34 & 102 & 1.00 & & 32 & 104 & 1.00 & \\
\hline & Dominant & $\mathrm{CC}-\mathrm{CT}$ & 112 & 252 & $1.32(0.83-2.07)$ & 0.239 & 94 & 270 & $1.09(0.68-1.75)$ & 0.725 \\
\hline & & TT & 34 & 102 & 1.00 & & 32 & 104 & 1.00 & \\
\hline & Recessive & CC & 35 & 90 & $0.87(0.55-1.38)$ & 0.545 & 35 & 90 & $1.13(0.70-1.82)$ & 0.617 \\
\hline & & CT-TT & 111 & 264 & 1.00 & & 91 & 284 & 1.00 & \\
\hline & Log-additive & - & - & - & $1.05(0.80-1.38)$ & 0.717 & - & - & $1.08(0.81-1.44)$ & 0.606 \\
\hline
\end{tabular}

CHD coronary heart disease, $\mathrm{Cl}$ confidence interval, $\mathrm{OR}$ odds ratio

$p$ values were calculated by unconditional logistic regression analysis with adjustment for age and gender; ${ }^{*} p<0.05$ indicates statistical significance

Table 8 Analysis of the association between clinical characteristics of diabetes patients and SNP genotypes

\begin{tabular}{|c|c|c|c|c|c|c|c|}
\hline SNP & FPG & $\mathrm{HbA1c}$ & $\mathrm{TC}(\mathrm{mmol} / \mathrm{L})$ & Urea (mmol/L) & Cys C & $\mathrm{LPa}(\mathrm{mg} / \mathrm{L})$ & $\mathrm{T} 4$ (ng/ml) \\
\hline \multicolumn{8}{|c|}{ rs3821236 } \\
\hline $\mathrm{AA}$ & $7.29 \pm 3.83$ & $7.35 \pm 3.40$ & $4.16 \pm 1.14$ & $6.15 \pm 2.16$ & $0.93 \pm 0.30$ & $255.50 \pm 262.67$ & $6.95 \pm 1.60$ \\
\hline$A G$ & $7.41 \pm 3.38$ & $7.94 \pm 1.99$ & $4.22 \pm 2.61$ & $6.69 \pm 4.13$ & $1.04 \pm 0.56$ & $209.67 \pm 214.89$ & $7.00 \pm 1.89$ \\
\hline GG & $7.29 \pm 3.03$ & $8.08 \pm 1.97$ & $4.13 \pm 1.10$ & $6.52 \pm 1.89$ & $1.02 \pm 0.33$ & $199.87 \pm 199.50$ & $6.82 \pm 1.83$ \\
\hline$p$ & 0.934 & 0.714 & 0.893 & 0.342 & 0.125 & 0.136 & 0.647 \\
\hline \multicolumn{8}{|c|}{ rs11893432 } \\
\hline GG & $7.21 \pm 3.74$ & $7.94 \pm 1.92$ & $4.19 \pm 1.19$ & $6.18 \pm 2.22$ & $0.94 \pm 0.31$ & $269.81 \pm 281.32$ & $6.91 \pm 1.68$ \\
\hline GC & $7.31 \pm 3.34$ & $8.12 \pm 2.15$ & $4.18 \pm 2.61$ & $6.65 \pm 4.12$ & $1.03 \pm 0.56$ & $205.84 \pm 204.67$ & $7.02 \pm 1.88$ \\
\hline CC & $7.53 \pm 3.21$ & $8.11 \pm 1.99$ & $4.17 \pm 1.09$ & $6.57 \pm 1.91$ & $1.02 \pm 0.33$ & $193.76 \pm 192.91$ & $6.83 \pm 1.79$ \\
\hline$p$ & 0.768 & 0.735 & 0.997 & 0.438 & 0.205 & $0.021^{*}$ & 0.657 \\
\hline \multicolumn{8}{|c|}{ rs11889341 } \\
\hline $\mathrm{TT}$ & $7.69 \pm 5.02$ & $8.03 \pm 1.92$ & $4.17 \pm 1.11$ & $6.28 \pm 2.38$ & $1.00 \pm 0.35$ & $259.67 \pm 257.00$ & $6.63 \pm 1.63$ \\
\hline $\mathrm{TC}$ & $7.17 \pm 3.30$ & $8.10 \pm 2.19$ & $4.23 \pm 2.64$ & $6.61 \pm 3.96$ & $0.98 \pm 0.45$ & $218.45 \pm 232.96$ & $6.90 \pm 1.70$ \\
\hline $\mathrm{CC}$ & $7.48 \pm 3.03$ & $8.05 \pm 1.91$ & $4.13 \pm 1.07$ & $6.47 \pm 2.36$ & $1.05 \pm 0.48$ & $204.18 \pm 198.44$ & $7.08 \pm 1.97$ \\
\hline$p$ & 0.512 & 0.969 & 0.872 & 0.787 & 0.246 & 0.318 & 0.298 \\
\hline \multicolumn{8}{|c|}{ rs 7574865} \\
\hline $\mathrm{TT}$ & $6.94 \pm 2.80$ & $8.07 \pm 1.98$ & $4.17 \pm 1.07$ & $6.34 \pm 2.42$ & $1.09 \pm 0.83$ & $244.93 \pm 253.88$ & $6.52 \pm 1.56$ \\
\hline $\mathrm{TG}$ & $7.39 \pm 3.78$ & $8.13 \pm 2.20$ & $4.22 \pm 2.63$ & $6.58 \pm 3.92$ & $0.96 \pm 0.30$ & $218.35 \pm 233.32$ & $6.94 \pm 1.72$ \\
\hline GG & $7.39 \pm 3.01$ & $8.00 \pm 1.90$ & $4.14 \pm 1.11$ & $6.48 \pm 2.44$ & $1.05 \pm 0.49$ & $208.48 \pm 200.50$ & $7.05 \pm 1.96$ \\
\hline$p$ & 0.725 & 0.823 & 0.901 & 0.874 & $0.033^{*}$ & 0.618 & 0.246 \\
\hline \multicolumn{8}{|c|}{ rs897200 } \\
\hline CC & $7.23 \pm 3.00$ & $8.21 \pm 2.09$ & $4.09 \pm 1.00$ & $6.35 \pm 2.46$ & $1.01 \pm 0.38$ & $212.91 \pm 216.65$ & $7.26 \pm 1.93$ \\
\hline $\mathrm{CT}$ & $7.53 \pm 3.57$ & $8.07 \pm 2.09$ & $4.10 \pm 1.10$ & $6.76 \pm 4.63$ & $0.99 \pm 0.55$ & $209.38 \pm 214.61$ & $6.99 \pm 1.90$ \\
\hline $\mathrm{TT}$ & $7.14 \pm 3.45$ & $7.96 \pm 1.98$ & $4.41 \pm 3.44$ & $6.76 \pm 4.63$ & $1.04 \pm 0.32$ & $233.92 \pm 242.07$ & $6.55 \pm 1.44$ \\
\hline$p$ & 0.558 & 0.628 & 0.297 & 0.502 & 0.698 & 0.600 & $0.010^{*}$ \\
\hline
\end{tabular}

HbA1c glycosylated hemoglobin, TC total cholesterol, FPG fasting plasma glucose, CysC: cystatin C;

LPa: Iysophosphatidic acid;

Lpa: lipoprotein a;

T4: thyroxine;

${ }^{*} p<0.05$ indicates statistical significance 
Table 9 SNP-SNP interaction models analyzed by the MDR method

\begin{tabular}{|c|c|c|c|c|c|}
\hline Model & Training Bal. Acc & Testing Bal. Acc & CVC & OR $(95 \% \mathrm{Cl})$ & $p$ \\
\hline rs3821236 & 0.532 & 0.515 & $9 / 10$ & $1.35(1.03-1.77)$ & $0.032^{*}$ \\
\hline rs3821236, rs897200 & 0.542 & 0.523 & $5 / 10$ & $1.38(1.08-1.77)$ & $0.011^{*}$ \\
\hline $\begin{array}{l}\text { rs3821236, rs11889341, } \\
\text { rs } 897200\end{array}$ & 0.555 & 0.499 & $5 / 10$ & 1.52(1.19-1.96) & $0.001^{*}$ \\
\hline $\begin{array}{r}\text { rs3821236, rs1 1893432, } \\
\text { rs11889341, rs897200 }\end{array}$ & 0.572 & 0.496 & $8 / 10$ & $1.73(1.35-2.22)$ & $<0.0001^{*}$ \\
\hline $\begin{array}{l}\text { rs3821236, rs11893432, } \\
\text { rs11889341, rs7574865, } \\
\text { rs897200 }\end{array}$ & 0.579 & 0.501 & $10 / 10$ & $1.85(1.43-2.38)$ & $<0.0001^{*}$ \\
\hline
\end{tabular}

MDR multifactor dimensionality reduction, Bal. Acc. balanced accuracy, CVC cross-validation consistency, OR odds ratio,

$\mathrm{Cl}$ : confidence interval;

$p$ values were calculated using $x^{2}$ tests, ${ }^{*} p<0.05$ indicates statistical significance

\section{Abbreviations}

STAT4: Signal Transcription and Transducer 4; T2D: Type 2 diabetes; SNPs: Single nucleotide polymorphisms; PCA: Principal component analysis; OR: Odds ratio; Cl: Confidence interval; MDR: Multifactor dimensionality reduction; MAF: Minor allele frequency; SLE: Systemic lupus erythematosus.

\section{Supplementary Information}

The online version contains supplementary material available at https://doi. org/10.1186/s12920-021-01000-2.

Additional file 1: Figure 1 Principal component analysis based on genotyping data of 1001 participants. The distance of each sample on the horizontal and vertical axes represents the similarity distance influenced by the principal component. The stronger the relevance of participants, the closer they are in PCA; the weaker the relevance of participants, the more scattered they are in PCA.

Additional file 2: Figure 2 Heat map of kinship matrix for1001 participants. The color of each lattice represents the correlation between rows and columns. The more red the color is, the stronger positive correlation is; and the more blue the color is, the stronger negative correlation is.

Additional file 3: Table Genotyping results of all participants.

\section{Acknowledgements}

We thank all authors for their contributions and supports. We are also grateful to all participants for providing blood samples.

\section{Authors' contributions}

The work presented here was carried out in collaboration between all authors. $\mathrm{JC}$ and RT carried out the molecular genetic studies and drafted the manuscript; JX, YT and JP designed the methods and experiments; NW, HC and JY performed the statistical analyses and interpreted the results. YP designed primers. S F, CG and WL performed the SNP genotyping experiments. JC and RT worked on associated data collection and their interpretation. WC conceived of the study, participated in the design and coordination of the study. All authors read and approved the final manuscript.

\section{Funding}

This work was supported by the Natural Science Foundation of Shaanxi Province (No. 2020GXLH-Y-029), the Bethune-Merck Diabetes Research Foundation (No. G-X-2019-056), the Clinical Research Award of the First Affiliated Hospital of Xi'an Jiaotong University, China (No. XJTU1AF-CRF-2019-007)

\section{Availability of data and materials}

The datasets supporting the conclusions of this article are included within the article and its Additional file 3.

\section{Declarations}

\section{Ethics approval and consent to participate}

This study was conducted under the standard approved by the First Affiliated Hospital of Xi'an Jiaotong University, and conformed to the ethical principles for medical research involving humans of the World Medical Association Declaration of Helsinki. All participants signed informed consent forms before participating in this study.

\section{Consent to publication}

Not Applicable.

\section{Competing interests}

The authors declare that they have no competing interest.

\section{Author details}

'Department of Endocrinology and Second Department of Geriatrics, The First Affiliated Hospital of Xi'an Jiaotong University, \#277 West Yanta Road, Xi'an 710061, Shaanxi, China. ${ }^{2}$ Department of Oncology, East Branch of The First Affiliated Hospital of Xi'an Jiaotong University, Xi'an 710089, Shaanxi, China. ${ }^{3}$ Department of Endocrinology, Xianyang Central Hospital, Xianyang 712000 , Shaanxi, China.

Received: 19 May 2021 Accepted: 4 June 2021

Published online: 27 June 2021

\section{References}

1. Meigs JB, Cupples LA, Wilson PW. Parental transmission of type 2 diabetes: the Framingham Offspring Study. Diabetes. 2000;49(12):2201-7.

2. Kaprio J, Tuomilehto J, Koskenvuo M, Romanov K, Reunanen A, Eriksson J, et al. Concordance for type 1 (insulin-dependent) and type 2 (non-insulin-dependent) diabetes mellitus in a population-based cohort of twins in Finland. Diabetologia. 1992;35(11):1060-7.

3. Mills JL, Irving RR, Choo-Kang EG, Wright-Pascoe R, McLaughlin W, Mullings AA, et al. Multigenerational inheritance and clinical characteristics of three large pedigrees with early-onset type 2 diabetes in Jamaica. Pan Am J Public Health. 2010;27(6):435-41.

4. Schmidt MI, Duncan BB, Sharrett AR, Lindberg G, Savage PJ, Offenbacher $\mathrm{S}$, et al. Markers of inflammation and prediction of diabetes mellitus in adults (Atherosclerosis Risk in Communities study): a cohort study. Lancet (London, England). 1999:353(9165):1649-52.

5. Thomas F, Balkau B, Vauzelle-Kervroedan F, Papoz L. Maternal effect and familial aggregation in NIDDM. The CODIAB Study. CODIAB-INSERMZENECA Study Group. Diabetes. 1994;43(1):63-7.

6. Huang X, Wang Z, Jia N, Shangguan S, Lai J, Cui X, et al. Association between STAT4 polymorphisms and the risk of juvenile idiopathic arthritis in Han Chinese populations. Clin Exp Rheumatol. 2019;37(2):333-7. 
7. Chang HC, Zhang S, Kaplan MH. Neonatal tolerance in the absence of Stat4- and Stat6- dependent Th cell differentiation. J Immunol. 2002;169(8):4124-8.

8. Chitnis T, Salama AD, Grusby MJ, Sayegh MH, Khoury SJ. Defining Th1 and Th2 immune responses in a reciprocal cytokine environment in vivo. J Immunol. 2004;172(7):4260-5

9. Cunnion KM, Krishna NK, Pallera HK, Pineros-Fernandez A, Rivera MG, Hair PS, et al. Complement activation and STAT4 expression are associated with early inflammation in diabetic wounds. PLOS ONE. 2017;12(1):e0170500.

10. Zhao R, Sun Y, Zhang Y, Wang W, Wang S, Wang C, et al. Distinguishable immunologic characteristics of COVID-19 patients with comorbid type 2 diabetes compared with nondiabetic individuals. Mediat Inflamm. 2020;2020:6914878

11. Mahlangu T, Dludla PV, Nyambuya TM, Mxinwa V, Mazibuko-Mbeje SE, Cirilli I, et al. A systematic review on the functional role of Th1/Th2 cytokines in type 2 diabetes and related metabolic complications. Cytokine. 2020;126:154892.

12. Nekoua MP, Fachinan R, Atchamou AK, Nouatin O, Amoussou-Guenou D. Amoussou-Guenou MK, et al. Modulation of immune cells and Th1/ Th2 cytokines in insulin-treated type 2 diabetes mellitus. Afr Health Sci. 2016;16(3):712-24.

13. Barton A, Thomson W, Ke X, Eyre S, Hinks A, Bowes J, et al. Re-evaluation of putative rheumatoid arthritis susceptibility genes in the post-genome wide association study era and hypothesis of a key pathway underlying susceptibility. Hum Mol Genet. 2008;17(15):2274-9.

14. Harley JB, Alarcon-Riquelme ME, Criswell LA, Jacob CO, Kimberly RP, Moser KL, et al. Genome-wide association scan in women with systemic lupus erythematosus identifies susceptibility variants in ITGAM, PXK KIAA1542 and other loci. Nat Genet. 2008;40(2):204-10.

15. Kobayashi S, Ikari K, Kaneko H, Kochi Y, Yamamoto K, Shimane K, et al. Association of STAT4 with susceptibility to rheumatoid arthritis and systemic lupus erythematosus in the Japanese population. Arthritis Rheum. 2008;58(7):1940-6.

16. Orozco G, Alizadeh BZ, Delgado-Vega AM, Gonzalez-Gay MA, Balsa A, Pascual-Salcedo D, et al. Association of STAT4 with rheumatoid arthritis: a replication study in three European populations. Arthritis Rheum. 2008:58(7):1974-80
17. Palomino-Morales RJ, Rojas-Villarraga A, Gonzalez Cl, Ramirez G, Anaya JM, Martin J. STAT4 but not TRAF1/C5 variants influence the risk of developing rheumatoid arthritis and systemic lupus erythematosus in Colombians. Genes Immun. 2008;9(4):379-82.

18. Remmers EF, Plenge RM, Lee AT, Graham RR, Hom G, Behrens TW, et al. STAT4 and the risk of rheumatoid arthritis and systemic lupus erythematosus. N Engl J Med. 2007;357(10):977-86.

19. Mirkazemi S, Akbarian M, Jamshidi AR, Mansouri R, Ghoroghi S, Salimi Y, et al. Association of STAT4 rs7574865 with susceptibility to systemic lupus erythematosus in Iranian population. Inflammation. 2013;36(6):1548-52.

20. Yang J, Lee SH, Goddard ME, Visscher PM. GCTA: a tool for genome-wide complex trait analysis. Am J Hum Genet. 2011;88(1):76-82.

21. Dai ZJ, Liu XH, Ma YF, Kang HF, Jin TB, Dai ZM, et al. Association between single nucleotide polymorphisms in DNA polymerase kappa gene and breast cancer risk in Chinese Han population: a STROBE-compliant observational study. Medicine. 2016;95(2):e2466.

22. Ignacio A, Breda CNS, Camara NOS. Innate lymphoid cells in tissue homeostasis and diseases. World J Hepatol. 2017;9(23):979-89.

23. Zhou TB, Jiang ZP, Qin YH, Zhou JF. Retracted Association of STAT4 gene polymorphism with systemic lupus erythematosus/lupus nephritis risk. Nephrology (Carlton, Vic). 2014.

24. Carmona FD, Onat AM, Fernandez-Aranguren T, Serrano-Fernandez A, Robledo G, Direskeneli H, et al. Analysis of systemic sclerosis-associated genes in a Turkish population. J Rheumatol. 2016;43(7):1376-9.

25. Thompson SD, Sudman M, Ramos PS, Marion MC, Ryan M, Tsoras M, et al. The susceptibility loci juvenile idiopathic arthritis shares with other autoimmune diseases extend to PTPN2, COG6, and ANGPT1. Arthritis Rheum. 2010;62(11):3265-76.

26. Fletcher B, Gulanick M, Lamendola C. Risk factors for type 2 diabetes mellitus. J Cardiovasc Nurs. 2002;16(2):17-23.

27. Li C, Zhao L, Wang W, Li H, Meng X, Chen S. STAT4 rs7574865 polymorphism contributes to the risk of type 1 diabetes: a meta analysis. Int J Clin Exp Med. 2015;8(2):2471-5.

\section{Publisher's Note}

Springer Nature remains neutral with regard to jurisdictional claims in published maps and institutional affiliations. 